\title{
Mindestlöhne und Beschäftigung - Die theoretische Debatte und empirische Ergebnisse
}

Viele Länder haben ihn schon seit Langem und flächendeckend: den Mindestlohn. Für Deutschland gilt dies erst seit Kurzem und nur in wenigen Branchen. Und nach Auffassung einiger Politiker und Ökonomen wäre es sogar besser, gar keinen Mindestlohn zu haben. Er - so das Argument der Kritiker - vermindere Beschäftigung und verfestige Arbeitslosigkeit; dies zeige sowohl die Theorie wie auch die praktische Erfahrung. Untersucht man diese Argumentation allerdings näher, so zeigt sich, dass weder Theorie noch empirische Forschung einen eindeutigen Beleg für diese Aussage liefern können. Insbesondere in Deutschland spricht wenig dafür, dass ein moderater Mindestlohn sich negativ auf die Beschäftigung auswirken würde. ${ }^{1}$

\section{Einleitung}

Erst kürzlich hat der Deutsche Gewerkschaftsbund (DGB) seine Forderung nach einem flächendeckenden Mindestlohn von 7,50€ auf 8,50€ erhöht. Für eine gesetzliche Lohnuntergrenze spricht er sich bereits seit 2004 aus. Diese Forderung sei, so der DGB, vor allem dem drastischen Anwachsen des Niedriglohnsektors in Deutschland innerhalb der letzten Jahre geschuldet. Ein Mindestlohn soll unter anderem die schlimmsten Auswüchse dieses Phänomens bekämpfen und die Aufnahme einer Beschäftigung lohnenswerter machen. Ökonomen stehen dieser Forderung oftmals kritisch gegenüber und verweisen auf die zu erwartenden negativen Beschäftigungseffekte. Im Zuge der lauter werdenden Appelle nach einer Lohnuntergrenze und der gestiegenen Bereitschaft der Regierung, dieses wirtschaftspolitische Mittel zur Gestaltung der Rahmenbedingungen zumindest in einigen Sektoren aktiv einzusetzen, produzierten angesehene deutsche Forschungsinstitute eine Reihe von Berechnungen, die den Beschäftigungseffekt eines Mindestlohns zu quantifizieren versuchten. Entsprechende Prognosen bezogen auf einen Mindestlohn von 7,50€ - reichen von einigen hunderttausend bis zu 1,2 Mio. verlorenen Arbeitsplätzen. Damit haben Mindestlohngegner in der politischen Diskussion alarmierende und scheinbar wissenschaftlich fundierte Argumente. Jedoch: Diese Ergebnisse sollten hinterfragt, zumindest aber vor dem
Hintergrund gewertet werden, dass es sich um ex-ante Simulationen und nicht um empirisch nachgewiesene Effekte (ex-post) handelt. Für solche Simulationsrechnungen müssen die Wissenschaftler Annahmen treffen. Dass hierbei durchgehend negative Effekte errechnet werden, liegt vor allem an den zugrunde liegenden Modellen.

Grundsätzlich unterstellen die Forscher negative Elastizitätsparameter. Auch wenn einige der Prognosen Substitutionseffekte berücksichtigen, ist es somit weitestgehend vorherbestimmt, dass die Simulationen einen Verlust von Arbeitsplätzen durch Mindestlöhne zum Ergebnis haben (Müller 2009). Wenn man sich auf die Ergebnisse einer solchen Prognose beruft, sollten die zugrunde liegenden Annahmen durch Theorie und empirische Forschung gesichert sein. Das aber ist bei den Simulationsrechnungen zu den Effekten von Mindestlöhnen nicht der Fall. Auch wenn die Forscher in ihren Studien anerkennen, dass die theoretische Debatte offen ist, verweisen sie auf die ihres Erachtens klaren empirischen Ergebnisse der Mindestlohnforschung, um ihre Annahme von negativen Beschäftigungseffekten zu unterlegen. Eine der in jüngster Zeit in diesem Zusammenhang meist zitiertesten Quellen ist die Übersichtsstudie „Minimum Wages and Employment" von $\mathrm{Neu}$ mark und Wascher (2007), die bei einem Großteil der empirischen Untersuchungen negative Beschäftigungseffekte identifiziert haben (Knabe/Schöb 2008, S. 10).

Ob sich die Annahme, dass Mindestlöhne per se negative Beschäftigungswirkungen haben, aus der theoretischen Dis- kussion oder der empirischen Forschung tatsächlich ableiten lässt und man sich deshalb von der Idee eines Mindestlohnes verabschieden sollte, ist jedoch keineswegs ausgemacht. Dieser Artikel wird im Folgenden die wichtigsten theoretischen Modelle erörtern und veranschaulichen, dass ihre Annahmen bezüglich der Beschäftigungseffekte von Mindestlöhnen weit weniger eindeutig sind als vielfach vorgetragen (Abschnitt 2). Danach wird ein kurzer Überblick über die Literatur zum empirischen Forschungsstand gegeben und insbesondere auf die Übersichtsstudie von Neumark und Wascher (2007) eingegangen. Es kann gezeigt werden, dass diese Arbeit erhebliche methodische Schwächen hat und dass auch die empirische Forschung insgesamt keine eindeutigen und belastbaren Ergebnisse zum Effekt von Mindestlöhnen liefern kann (Abschnitt 3). Abschließend wird anhand der gewonnenen Erkenntnisse eine kurze Einschätzung zur potenziellen Wirkung eines Mindestlohnes in Deutschland gegeben (Abschnitt 4).

\footnotetext{
Der Verfasser dankt Miriam Beblo, Hansiörg Herr und zwei anonymen Gutachtern der WSIMitteilungen für ihre hilfreichen Kommentare und wertvollen Anregungen zu einer früheren Fassung dieses Beitrags.
}

Daniel Detzer, Student im Master-Programm "International Economics" der Hochschule für Wirtschaft und Recht (HWR) in Berlin. Arbeitsschwerpunkte: Nationale und internationale Makroökonomie, Wachstums- und Verteilungstheorie.

e-mail: danieldetzer@aol.com 


\section{Mindestlöhne in der ökonomischen Theorie}

Befragt man Ökonomen zum theoretischen Effekt von Mindestlöhnen, so können die Antworten je nach herangezogenem Modell und Paradigma unterschiedlich ausfallen.

\subsection{MINDESTLÖHNE IN DER NEOKLASSISCHEN THEORIE}

Wird das neoklassische Standardmodell eines perfekten Arbeitsmarktes herangezogen, ist das Ergebnis einfach darzustellen: Ohne einen Mindestlohn stellen Arbeitgeber einen Arbeitsuchenden ein, sofern der von ihm geforderte Lohn unter seiner Grenzproduktivität liegt. Die Arbeitnehmer wiederum sind solange bereit zu arbeiten, solange die gebotenen Löhne über ihrem subjektiv bestimmten Wert für alternative Aktivitäten, wie z.B. Freizeit, liegen. Es bildet sich ein gleichgewichtiger Lohnsatz, bei dem es keine unfreiwillig Arbeitslosen mehr gibt und bei dem auch die Arbeitgeber alle Stellen besetzen können. Ein Mindestlohn, der unter diesem Gleichgewichtslohn liegt, wird nach diesem Modell keinen Effekt haben. Ein Mindestlohn über dem Gleichgewichtslohn sorgt dafür, dass die Bezahlung einiger Arbeitnehmer höher ist als deren Ertrag für das Unternehmen. Ein ökonomisch denkender Arbeitgeber wird diese nun entlassen. Gleichzeitig wird aber durch den über dem Gleichgewichtslohn liegenden Mindestlohn eine zusätzliche Anzahl von erwerbsfähigen, aber bis dato nicht erwerbstätigen Personen motiviert, sich einen Job zu suchen. Es kommt zu unfreiwilliger Arbeitslosigkeit: zum einen durch die Arbeitgeber, die weniger Stellen anbieten, zum anderen durch die zusätzlich auf den Markt stoßenden Arbeitnehmer (Herr 2002, S. 7ff.).

Folgt man diesen Annahmen, so wird verständlich, weshalb Ökonomen, die dieses Modell für ihre Prognosen nutzen, einen Mindestlohn ablehnen. Doch das Modell ist keineswegs unumstritten. Einige Ökonomen weisen darauf hin, dass die neoklassische Arbeitsnachfragefunktion auf einer makroökonomischen Ebene theoretisch nur schwer nachweisbar sei. Kritisiert werden die unterstellten konstanten Skaleneffekte, weil in der Realität oftmals eher steigende Skaleneffekte zu verzeichnen seien. Unterstellt man diese, könnte ein höherer Lohn sogar zu einer steigenden Arbeitsnachfrage führen (Herr et al. 2009, S. 18).

Ein weiterer Kritikpunkt ist die Form der Arbeitsangebotsfunktion. Wie bereits erwähnt, geht das Lehrbuchmodell davon aus, dass Haushalte ihr Arbeitsangebot bei steigenden Löhnen ausweiten. Bei fallenden Löhnen aber würden sie sich entscheiden, weniger zu arbeiten. Eine alternative Denkweise, die ebenfalls mit der neoklassischen Theorie zu vereinbaren wäre und insbesondere im Niedriglohnsektor zutrifft, wäre, dass Haushalte ihr Arbeitsangebot bei einem sinkenden Lohnsatz ausweiten. Erklärt werden kann dies dadurch, dass, sobald das Einkommen eines Haushaltes unter eine bestimmte Höhe fällt, seine Mitglieder sich entscheiden, zusätzliche Stunden zu arbeiten oder einen zweiten Job anzunehmen, um zu überleben oder einen bestimmten Lebensstandard zu halten. Unter dieser Annahme ergibt sich eine Situation mit mehreren Gleichgewichten. Eines mit einer hohen Beschäftigung und niedrigen Löhnen und eines mit höheren Löhnen und geringerer Beschäftigung. In keinem der Gleichgewichte besteht unfreiwillige Arbeitslosigkeit. Aus rein ökonomischer Sichtweise kann nicht entschieden werden, welches dieser Gleichgewichte zu bevorzugen ist. Moralisch oder politisch gesehen ist jedoch ein Gleichgewicht mit niedrigen Löhnen und langen Arbeitszeiten zumindest zweifelhaft. Ein Mindestlohn würde es hier dem Gesetzgeber ermöglichen, das Niedriglohngleichgewicht zu verhindern (Prasch 2000, S. 688).

Doch auch wenn man die Nachfrageund Angebotsfunktion in ihrer neoklassischen Lehrbuchform für den Arbeitsmarkt akzeptiert, muss in Rechnung gestellt werden, dass die scheinbar eindeutigen Ergebnisse auf bestimmten, d.h. gesetzten Annahmen beruhen; lässt man einige davon fallen, so verändern sich auch die Befunde. Ersetzt man zum Beispiel die Annahme homogener Arbeit und unterscheidet zwischen gut ausgebildeten und ungelernten Arbeitnehmern, so entsteht die Möglichkeit zur Substitution. Verteuert ein Mindestlohn ungelernte Arbeitnehmer relativ zu gut ausgebildeten, so ersetzen manche Unternehmen ungelernte Arbeiter durch besser ausgebildete. Dadurch wird ein Teil der Beschäftigungsverluste ausgeglichen. Der negative Effekt fällt im Gesamten niedriger aus als im Standardmodell unterstellt (Zavodny 1998, S. 20f.). In einigen extremen Fällen kann der Effekt neutral sein (Card/Krueger 1995, S. 360).

Des Weiteren abstrahiert das Modell von jeglicher Marktmacht. Der klassische Gegenpart zum beschriebenen Lehrbuchmodell ist das Monopson. Es wird unterstellt, dass ein einzelner Arbeitgeber einer Vielzahl von Arbeitsuchenden gegenübersteht. Durch diese Marktmacht sei es ihm möglich, die Arbeitnehmer unter ihrer Grenzproduktivität zu entlohnen. Um dies zu erreichen und gleichzeitig seinen Gewinn zu maximieren, muss er allerdings die Beschäftigung niedriger halten als sie in einer Situation vollkommenen Wettbewerbs wäre. Ein Mindestlohn könne hier sowohl die Anzahl der Beschäftigten als auch deren Löhne erhöhen (Herr et al. 2009, S. 19ff.). Auch wenn das Modell eines einzelnen Arbeitgebers für Deutschland weitestgehend irrelevant ist, so kann man doch in Such- und Effizienzlohnmodellen Situationen aufzeigen, die zu qualitativ gleichen Ergebnissen führen (Card/Krueger 1995, S. 369ff.). ${ }^{2}$ Diese sind für eine entwickelte Volkswirtschaft wie Deutschland aber deutlich realistischer als das klassische Monopson (Ragacs 2002, S. 15). Zusätzlich können positive Beschäftigungseffekte eines Mindestlohnes nicht nur in einem Monopson, sondern auch in Modellen mit monopsonistischer Konkurrenz oder oligopsonistischen Marktstrukturen nachgewiesen werden (Bhaskar et al. 2002, S. 167ff.).

Deutlich wird: Innerhalb des neoklassischen Paradigmas basiert der theoretische Nachweis eines negativen Beschäftigungseffektes von Mindestlöhnen auf strikten Annahmen, die allerdings innerhalb der Wissenschaft strittig sind. Und selbst wenn man sie akzeptiert, lässt sich zeigen, dass mehrere Gleichgewichte existieren können und dass ein Mindestlohn Einfluss darauf hat, zu welchem Gleichgewicht eine Volkswirtschaft tendiert. Lässt man einige der Annahmen fallen, dann schält sich ein geringerer negativer Beschäftigungseffekt heraus als das Stan-

\footnotetext{
2 Die Suchtheorie geht davon aus, dass die Arbeitnehmer nicht über vollkommene Informationen über alle Beschäftigungsmöglichkeiten auf dem Markt verfügen. Effizienzlohnmodelle unterstellen, dass Arbeitnehmer höhere Löhne zahlen, um zu effizienterer Arbeit anzuhalten und Fluktuation zu vermeiden.
} 
dardmodell suggeriert - oder er verkehrt sich sogar ins Positive. Somit kann die neoklassische Theorie keine eindeutige Antwort auf die Frage liefern, wie sich ein Mindestlohn auf die Beschäftigung in Deutschland auswirken würde.

\subsection{MINDESTLÖHNE IN DER KEYNESIANISCHEN THEORIE}

Geht man über die orthodoxe Theorie hinaus und betrachtet alternative Ansätze, fallen die Voraussagen ähnlich vielfältig aus. Anders als in der neoklassischen findet sich in der keynesianischen Theorie kein direkter Zusammenhang zwischen Löhnen und Beschäftigung. Vielmehr wird die Arbeitsnachfrage vom Produktionsvolumen, welches von der aggregierten Nachfrage abhängt, und der eingesetzten Technologie bestimmt. Unterstellt man zunächst homogene Arbeit, so kann ein Mindestlohn in der keynesianischen Theorie den Reallohn der Arbeitnehmer nicht verändern. Steigende Lohnkosten veranlassen die Unternehmen, die Preise in gleichem Maße zu erhöhen, sodass trotz gestiegener Nominallöhne die Kaufkraft der Arbeitnehmer unverändert bleibt. Geht man dagegen von heterogener Arbeit aus, so führt ein Mindestlohn, der die unteren Lohnklassen anhebt, dazu, dass die betroffenen Industrien einen Technologiewechsel vollziehen. Dadurch verändern sich deren Faktornachfrage und Produktpreise, was wiederum zu veränderter Nachfrage und Beschäftigung in vor- und nachgelagerten Industrien führt. Als Folge dieser Transmission kommt es letztlich in der gesamten Volkswirtschaft zu einer Veränderung der Nachfrage, des Angebots, der Preisstruktur und der Arbeitsnachfrage. Aufgrund dieser vielfältigen Einflüsse und Variablen vermag auch die keynesianische Theorie nicht zu sagen, wie sich ein Mindestlohn auf die Gesamtnachfrage nach Arbeit auswirkt. Der Effekt kann positiv, negativ oder auch neutral sein (Herr et al. 2009, S. 11f.). Wenn ein Mindestlohn allerdings Einkommen von den oberen zu den unteren Lohnklassen umverteilt, also die Lohnstruktur von unten staucht, so sagt die Theorie voraus, dass es zu einer Steigerung der aggregierten Nachfrage kommt, welche als essenziell für mehr Wachstum und Beschäftigung angesehen wird (Bartsch 2007, S. 14f.).

Zusammenfassend ist festzuhalten, dass weder die neoklassische noch die keynesianische Theorie klare Aussagen über den Effekt eines Mindestlohnes treffen kann. Das heißt aber auch, dass der Vorwurf der Gegner eines Mindestlohns, Mindestlohnbefürworter "leugnen die einfachsten ökonomischen Zusammenhänge“ (Sinn 2007), ins Leere läuft.

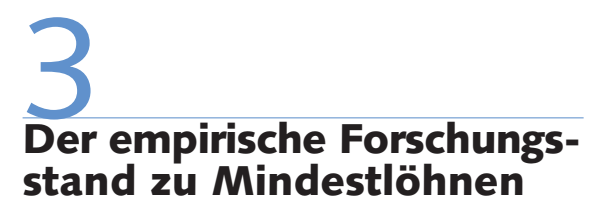

\subsection{EIN ÜBERBLICK}

Wenn in der Ökonomie theoretische Debatten keine befriedigenden Antworten liefern können, werden oftmals Ergebnisse empirischer Forschung zu Hilfe genommen. Infolgedessen haben viele Forscher versucht, den Effekt mithilfe von ökonometrischen Verfahren zu bestimmen. ${ }^{3}$ Die frühen Studien, die bis zum Ende der 1970er Jahre entstanden sind, weisen klar auf negative Beschäftigungseffekte hin (Ragacs 2004, S. 31). Brown et al. (1982, S. 534f.) haben die Ergebnisse dieser ersten Forschungswelle zusammengefasst. Sie kamen zu dem Befund, dass eine Erhöhung des Mindestlohnes um $10 \%$ zu einem Beschäftigungsverlust von $1 \%$ bis $3 \%$ bei jugendlichen Arbeitnehmern führt. Für junge Erwachsene konstatierten sie ebenfalls einen, wenn auch kleineren, negativen Effekt. Aussagen über die Auswirkung eines Mindestlohnes auf die Beschäftigungschancen von Erwachsenen erlaubte das ihnen vorliegende Forschungsmaterial dagegen nicht.

Auf diesem Stand kam die Mindestlohnforschung zunächst ins Stocken und der Befund eines Beschäftigungsverlustes von $1 \%$ bis $3 \%$ durch einen Mindestlohn wurde gemeinhin akzeptiert und wiederholt in politischen Debatten gegen die Festlegung von Lohnuntergrenzen vorgebracht (Card/Krueger 1995, S. 180). Angeregt durch unterschiedliche Mindestlohngesetze in einigen Staaten der USA startete in den 1980er Jahren eine neue Forschungswelle. Diese wird häufig als der „New Minimum Wage Research“ bezeichnet (Neumark/Wascher 2008, S. 2). In diesen neuen Untersuchungen sind die Ergebnisse weniger eindeutig. Neben negativen Effekten, die einige Studien ausweisen, konnten viele Studien keinen Effekt ausmachen. Innerhalb dieser zweiten For- schungswelle ist die Arbeit von Card und Krueger (1995) ein besonderer Meilenstein (Herr et al. 2009, S. 2). Sie stellen die herkömmliche Sichtweise eines negativen Effektes von Mindestlöhnen infrage. Hierbei stützen sie sich auf einige methodologische Überlegungen, insbesondere bezüglich des häufig genutzten Zeitreihenverfahrens. Sie kritisieren vor allem Schwächen bei der Spezifikation der Modelle und der Auswahl der Variablen. Auch überprüfen sie die älteren Studien nochmals anhand aktuellerer Daten und kommen damit zu dem Ergebnis, dass sich nur noch geringfügige und statistisch unsignifikante Effekte von Mindestlöhnen identifizieren lassen. Kern ihres Buches sind aber vor allem auch ihre eigenen empirischen Studien. In diesen fanden sie nur insignifikante oder sogar positive Beschäftigungseffekte (Card/Krueger 1995). Hierdurch wurde eine neue Debatte ausgelöst - und viele Ökonomen änderten ihre Meinung über Mindestlöhne. ${ }^{4}$ Vehemente Mindestlohngegner aber zogen daraufhin mit eigenen neuen Untersuchungen ins Feld und kritisierten die Ergebnisse von Card/Krueger. Insbesondere die Ökonomen Neumark und Wascher sind hier zu nennen, die seitdem mit verschiedenen Übersichtsstudien über die vorhandene Mindestlohnliteratur den Beweis für die negativen Beschäftigungseffekte eines Mindestlohns zu erbringen suchen. $\mathrm{Ob}$ dies gelungen ist, ist Thema des folgenden Abschnitts.

\subsection{NEUMARK UND WASCHER - BELEG FÜR EINEN NEGATIVEN BESCHÄFTIGUNGSEFFEKT?}

Den letzten und umfangreichsten Überblick über empirische Forschungsbefunde zu den Effekten von Mindestlöhnen veröffentlichten Neumark und Wascher vor drei Jahren (Neumark/Wascher 2007). Sie untersuchen insgesamt 102 Mindestlohnstudien daraufhin, ob die jeweiligen Untersuchungen einen Beleg für einen positiven oder einen negativen Beschäftigungseffekt liefern. Insgesamt kommen sie zu dem Ergebnis, dass im Vergleich zu

\footnotetext{
3 Kritisch ist hier zu bemerken, dass es sich bei den Analysen bezüglich der Wirkung eines Mindestlohns häufig um Partialanalysen des Arbeitsmarktes handelt.

4 Zur neueren empirischen Mindestlohnforschung vgl. auch den Beitrag von Gerhard Bosch in diesem Heft.
} 
den Studien der ersten Forschungswelle, die Schätzungen über den Effekt eines Mindestlohns weiter auseinander gehen. Sie reichen von weit unter -1 bis weit über 0 . Das bedeutet, dass neben negativen Beschäftigungseffekten auch positive Effekte gefunden wurden. Diese Diskrepanz wird in der Studie damit begründet, dass in der zweiten Forschungswelle deutlich mehr Daten zur Verfügung standen und neuere empirische Methoden eingesetzt werden konnten. Entscheidender für die politische Diskussion erscheint aber ihr Urteil, dass die neuere Mindestlohnforschung keineswegs die konventionelle Sichtweise widerlegen würde, dass Mindestlöhne einen negativen Beschäftigungseffekt hätten. Die von ihnen ausgewerteten Studien lieferten zu zwei Dritteln Hinweise auf einen negativen Effekt von Mindestlöhnen und nur acht Studien würden auf einen positiven Effekt verweisen. Um ihr Argument gegen Mindestlöhne zu verstärken, wählen die Autoren Studien aus, die sie als am zuverlässigsten einstufen. Von diesen 33 Studien weisen sogar $85 \%$ auf einen negativen Effekt hin (ebd., S. 120ff.).

Bei einer oberflächlichen Betrachtung erweckt diese Übersichtsstudie den Eindruck, dass der empirische Beweis zur Schädlichkeit von Mindestlöhnen nunmehr erbracht sei. Beschäftigt man sich allerdings näher mit der Arbeit, wird deutlich, dass ein solcher Schluss auf der Grundlage der von ihnen gebotenen Ergebnisse, der einbezogenen Studien und der Vorgehensweise der Autoren voreilig ist.

(1) Zunächst fällt auf, dass ein Großteil der Studien sich explizit mit dem Effekt von Mindestlöhnen auf die Beschäftigung von Jugendlichen (Teenagern) beschäftigt. Dies liegt vor allem daran, dass diese aufgrund ihrer relativ niedrigen Produktivität und der entsprechend geringen Bezahlung mit einer höheren Wahrscheinlichkeit von einer Lohnuntergrenze betroffen sind und es deshalb leichter ist, einen ökonometrisch messbaren Effekt zu finden (Belchamber 2004, S. 31). Dies hat allerdings den Nachteil, dass, falls ein Effekt gefunden wird, dieses Ergebnis zunächst auch nur für diese Gruppe zutrifft. Im Weiteren muss man beachten, dass in den meisten Studien keine Substitutionseffekte berücksichtigt wurden. Das heißt, wenn sich ein Mindestlohn negativ auf die Beschäftigung von Teenagern auswirkt, dafür aber mehr junge Erwachsene eingestellt werden, überzeich- net eine Studie, die nur den Effekt auf die erste Gruppe misst, den gesamten Beschäftigungseffekt. Dolado et al. (1996, S. 352) finden in einer Studie zum spanischen Mindestlohn genau solch einen Effekt. Sie stellen fest, dass der dortige Mindestlohn die Beschäftigung in der Gruppe der Teenager gesenkt hat, sich gleichzeitig aber positiv auf die Beschäftigung anderer Bevölkerungsgruppen ausgewirkt hat. Pereira (2003, S. 8) identifiziert einen ähnlichen Effekt bei der Anhebung des Mindestlohns in Portugal.

(2) Neben diesem allgemeinen, bezüglich der herangezogenen Studien auftretenden Problem sollte man sich ebenfalls darüber bewusst sein, dass Neumark und Wascher Mindestlöhnen grundsätzlich kritisch gegenüberstehen (Fox 2006). Dies spiegelt sich auch in ihrer Übersichtstudie wider. Wenn man die Bewertungen der untersuchten Studien durch die Autoren mit den ursprünglichen Ergebnissen vergleicht, fällt auf, dass diese teilweise voneinander abweichen. Einige der Ergebnisse werden uminterpretiert, sodass sie als Bestätigung für einen negativen Effekt gelten, oder aber so dargestellt, dass ein unbedachter Leser den Eindruck gewinnt, der ursprüngliche Autor hätte einen negativen Effekt gefunden. Das Ergebnis der Studie von Dolado et al. (1996) zu Spanien stellen Neumark/ Wascher beispielsweise folgendermaßen dar: „The authors conclude from their study that minimum wage increases in Spain reduced youth employment" (Neumark/Wascher 2007, S. 101). Das Fazit in der Originalstudie lautete demgegenüber: „To conclude, we do have evidence from Spain that rising minimum wages in the early 1990s have reduced youth employment. But the evidence also suggests a rise in total employment. One should not conclude that the minimum wage must necessarily be bad for the labor market" (Dolado et al. 1996, S. 352). Der zweite Teil des Fazits wurde in der Studie von Neumark/Wascher nur in einer Fußnote erwähnt. Ein weiteres Beispiel für diese etwas fragwürdige Vorgehensweise findet sich anhand einer weiteren Untersuchung von Dolado et al. (1996, S. 352ff.) zur Abschaffung der britischen „Wage Councils“, welche bis dahin sektorspezifische Mindestlöhne festlegten. Während die Originalautoren keinen negativen Effekt durch deren Aktivität entdecken konnten, sahen Neumark/Wascher (2007, S. 81f.) diese Studie als Indiz für ei- nen negativen Effekt. Entsprechend sollte man sich beim Lesen der Überblicksstudie darüber im Klaren sein, dass die Autoren die Ergebnisse aus ihrer Sicht und teilweise in einer Weise interpretieren, die nicht immer mit der Einschätzung des Originalautors übereinstimmt.

(3) Auch die Auswahlkriterien für die Studien, die von Neumark/Wascher als besonders zuverlässig und aussagekräftig eingeschätzt werden, bleiben undurchsichtig. Sie werden nicht explizit benannt. Ersichtlich scheint eine grundlegende Skepsis gegenüber Fallstudien, was somit eines ihrer Kriterien sein könnte (Fox 2006). Und $20 \%$ der berücksichtigten Studien stammen von Neumark/Wascher selbst, was zwar ihr Vertrauen in die Validität ihrer eigenen Analysen zeigt, unter Objektivitätskriterien aber gleichwohl zu hinterfragen ist.

(4) Auch wenn die Übersichtsstudie mit 102 untersuchten Studien durchaus umfangreich ist, stellt sie bei Weitem keinen umfassenden Überblick über die gesamte empirische Literatur zu diesem Thema dar. Ein Großteil der rezipierten Studien befasst sich mit der Wirkung eines Mindestlohns in den USA (53 Studien). Die wachsende Anzahl an Untersuchungen aus anderen Ländern wurde von Neumark/ Wascher nur selektiv und vor allem nur dann zur Kenntnis genommen, wenn sie englischsprachig publiziert waren, was wiederum die Auswahl zugunsten der anglo-amerikanischen Länder verzerrt. Insgesamt flossen neben den bereits genannten 53 USA-Studien fünf Studien über Kanada, neun über Großbritannien, vier über Australien und drei über Neuseeland ein. Kontinentaleuropäische und skandinavische Länder sind in der Auswahl von Neumark/Wascher dagegen unterrepräsentiert - ein Umstand, der auch aus der Perspektive der Autoren als problematisch gewertet werden müsste, da sie selbst in einer anderen Arbeit (Neumark/ Wascher 2004) darauf hinweisen, dass sich unterschiedliche Arbeitsmarktinstitutionen auf den Beschäftigungseffekt von Mindestlöhnen auswirken. Hier stellen sie fest, dass ein Mindestlohn in Ländern mit geringem Kündigungsschutz und einer wenig aktiven Arbeitsmarktpolitik deutlich stärkere negative Effekte hat als in Volkswirtschaften, die über einen hohen Kündigungsschutz und eine aktive Arbeitsmarktpolitik verfügen (ebd., S. 242). 
Übersicht 1 zeigt, in welchen Ländern gemäß Neumark/Wascher Mindestlöhne besonders negativ und in welchen Ländern eher positiv wirken. Auffällig ist folgender Befund: Länder wie Deutschland mit einem relativ regulierten Arbeitsmarkt können positivere Wirkungen von einem Mindestlohn erwarten als z.B. die USA oder Großbritannien, die beide über Arbeitsmärkte mit relativ großen Flexibilitätsspielräumen verfügen. Dieser Befund widerspricht dem Argument der Mindestlohngegner, dass in Deutschland aufgrund seines wenig flexiblen Arbeitsmarktes der Effekt eines Mindestlohnes negativer ausfallen wird.

Zugleich veranschaulicht Übersicht 1, dass in der Überblicksstudie von Neumark/ Wascher eindeutig Länder überwiegen, in denen Mindestlöhne mit einer höheren Wahrscheinlichkeit negativ wirken. Entsprechend muss man beachten, dass die Ergebnisse dieser Untersuchung entweder nur auf Länder mit einem ähnlichen Institutionengefüge zutreffen können oder aber weniger eindeutig ausfallen würden, wenn eine ausgeglichenere Länderauswahl getroffen worden wäre.

\subsection{GIBT ES EINEN „PUBLIKATIONSBIAS“ IN DER MINDESTLOHNLITERATUR?}

Ein weiterer wichtiger Punkt, der mit Blick auf vorliegende empirische Studien zum Mindestlohn beachtet werden muss, wurde bereits 1995 von Card und Krueger angemerkt. Sie konstatieren für die früheren Mindestlohnstudien einen „Publikationsbias“. Das bedeutet, dass Fachzeitschriften dazu tendieren, Studien mit statistisch signifikanten Resultaten zu publizieren, während nicht signifikante Resultate weniger häufig veröffentlicht werden. Folglich sind empirische Untersuchungen, die keinen signifikanten Effekt feststellen können und somit die Argumentation von Mindestlohnbefürwortern unterstützen würden, in der zugänglichen Literatur unterrepräsentiert. Des Weiteren halten viele Ökonomen an der Vorstellung fest, dass sich Mindestlöhne negativ auf die Beschäftigung auswirken. Card und Krueger sind deshalb der Meinung, dass die Verfasser von Mindestlohnstudien, die eine gewisse Diskretion über die erklärenden Variablen haben, dazu neigen, die Modelle so zu spezifizieren, dass sie statistisch signifikante negative Ergebnisse erzielen. Mit statistischen Methoden ist es

\section{Übersicht 1: Der Effekt von Mindestlöhnen* in Abhängigkeit von verschiedenen Arbeitsmarktsituationen}

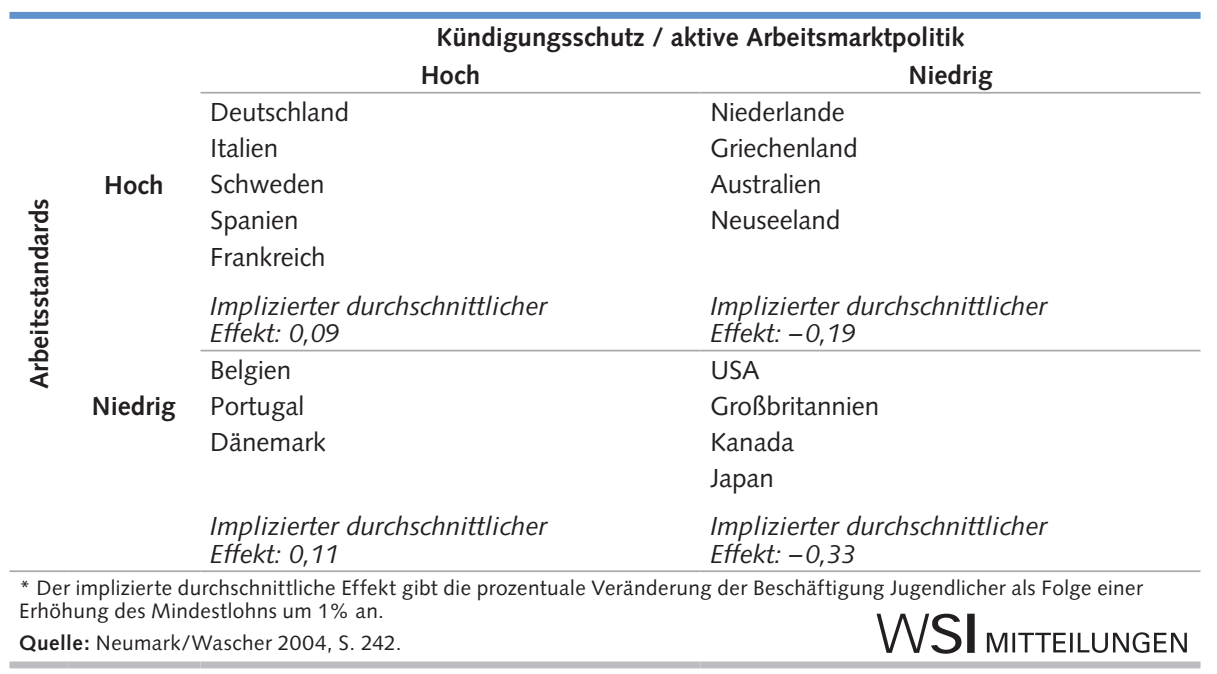

mittlerweile möglich, solche Vermutungen wissenschaftlich zu belegen. Card/Krueger versuchen dies anhand einer Metaanalyse von 15 Studien und finden tatsächlich Hinweise auf einen solchen Publikationsbias in der älteren Mindestlohnliteratur (Card/Krueger 1995, S. 186ff.). Neumark/ Wascher (1998, S. 468ff.) wiederholten die Analyse, konnten die Ergebnisse von Card und Krueger aber nicht bestätigen. Sie argumentieren, dass die Wirkung des Mindestlohnes im Zeitablauf nachgelassen habe. Solche strukturellen Veränderungen würden Card und Krueger ausblenden und mit einem Publikationsbias verwechseln.

Die Forscher Doucouliagos/Stanley (2009, S. 422f.) griffen die These eines Publikationsbias in der Mindestlohnliteratur erneut auf und replizierten die Studie mit neueren Methoden und insgesamt 64 Studien. Die Studien, die für diese Metaanalyse genutzt wurden, sind zu einem großen Teil identisch mit denen, die Neumark und Wascher für ihren Überblick genutzt haben (Doucouliagos/Stanley 2009, S. 416ff.).

Auch Doucouliagos/Stanley kritisieren Card und Krueger für einen Fehler in ihrer Metaanalyse, bestätigen aber im Grunde deren Aussage. Sie können klare Hinweise auf die bewusste Auswahl von Studien mit negativen Effekten in der Mindestlohnforschung finden. Wenn sie diese Verzerrung herausfiltern, kommen sie zu dem Ergebnis, dass die untersuchten Studien keine Hinweise auf einen negativen Beschäftigungseffekt mehr liefern. Sie finden sogar heraus, dass die negative Verzerrung größer ist als der durchschnittlich geschätzte negative Beschäftigungseffekt. Korrigiert man diese Verzerrung, so sprechen die vorliegenden Studien insgesamt eher dafür, dass Mindestlöhne einen kleinen positiven Effekt auf die Beschäftigung haben.

Um möglicher Kritik an ihren Ergebnissen vorzubeugen, diskutieren Doucouliagos/Stanley noch andere Faktoren, wie zum Beispiel die von Neumark/Wascher angeführten strukturellen Veränderungen, kommen aber auch hier zu dem Ergebnis, dass sogar unter sehr großzügigen Annahmen kein oder maximal ein nur sehr kleiner negativer Beschäftigungseffekt gefunden werden kann. Nimmt man die Bedenken ernst, die bezüglich des Publikationsbias innerhalb der Mindestlohnliteratur vorliegen, dann wird offensichtlich, dass der narrative Ansatz von Neumark/ Wascher zu kurz greift. Selbst wenn sie die Analyse nach bestem Wissen und Gewissen durchgeführt hätten, kann ihr Ergebnis nur so gut sein wie die zugrunde liegende Literatur. Da diese ein verzerrtes Bild zum Beschäftigungseffekt von Mindestlöhnen wiedergibt, kann die Analyse der beiden Forscher nur ähnlich verzerrt sein.

\subsection{ZWISCHENFAZIT}

Zusammenfassend lässt sich festhalten, dass die scheinbar eindeutigen Ergebnisse der frühen Mindestlohnforschung von der neueren Literatur in Zweifel gezogen werden. Die jüngsten Versuche von Mindestlohngegnern, diese zu zerstreuen, sind bisher gescheitert. Das gilt auch für die Überblicksstudie von Neumark und Wascher, weil ihre Analyse erhebliche Schwächen aufweist. Zum einen erscheint die Vorgehensweise 
der Autoren bei der Bewertung der Studien fragwürdig. Zum anderen zeigen sich deutliche Probleme bezüglich der genutzten Literatur. Diese ist stark auf Teenager und junge Erwachsene fokussiert. Weiterhin wird - bedingt durch den Sprachhorizont der Autoren - ein deutlich höheres Gewicht auf angelsächsisch geprägte Volkswirtschaften gelegt, was die Länderauswahl verzerrt. Eine weitere und noch gravierendere Verzerrung ergibt sich aufgrund eines feststellbaren Publikationsbias. Betrachtet man all die Schwächen der Studie zusammen, so wird ersichtlich, dass sie keinen glaubhaften Nachweis für einen negativen Effekt von Mindestlöhnen erbringen kann. Somit konnte auch diese äußerst umfangreiche Studie keinen Schlussstrich unter die Mindestlohndebatte ziehen und der Nachweis über die Richtung und die Höhe seines Beschäftigungseffekts ist weiterhin offen.

\section{1 Schlussfolgerungen}

Bleibt die Frage: Welche Erkenntnisse lassen sich gleichwohl aus der ökonomischen Forschung für die Mindestlohndiskussion ziehen? Festzuhalten ist, dass in den verschiedenen ökonomischen Paradigmen Löhne eine unterschiedliche Rolle spielen und sich deshalb auch ein Mindestlohn, je nach gewählter theoretischer Grundlage, anders auswirkt. Während in der keynesianischen Theorie der Effekt grundsätzlich offen ist, sagt das Standardmodell der orthodoxen Theorie zunächst negative Effekte vorher. Allerdings ist dieses Modell nicht unstrittig und es kann, ohne die grundlegenden Annahmen zu verändern, gezeigt werden, dass mehrere Gleichgewichte möglich sind, von denen manche aus politischer und sozialer Sicht weniger wünschenswert sind. Ein Mindestlohn würde hier Abhilfe schaffen. Sobald einige der restriktiven Annahmen des Standardmodells aufgegeben werden, lässt auch die neoklassische Theorie Fälle $\mathrm{zu}$, in denen ein Mindestlohn keinen oder sogar einen positiven Beschäftigungseffekt haben kann. Innerhalb von Effizienzlohnund Suchmodellen lassen sich die qualitativen Ergebnisse in verschiedenen durchaus realistischen Szenarien replizieren. Somit zeigt sich, dass die Mindestlohndebatte weder ein eindeutiges Pro noch ein eindeutiges Kontra aus der ökonomischen Theorie ableiten kann.
Nicht viel anders sieht das Resümee mit Blick auf die empirischen Forschungsbefunde zur Wirkung von Mindestlöhnen aus: Wenngleich die frühe empirische Forschung scheinbar eindeutig negative Effekte identifizierte, wurden diese Ergebnisse aufgrund methodischer Schwächen durch die neuere Forschung in Zweifel gezogen. Solche Vorbehalte gelten auch, wie in diesem Beitrag gezeigt wurde, gegenüber der von Mindestlohngegnern vielfach zitierten Studie von Neumark und Wascher. Und selbst wenn man ihrer Argumentation folgt, bleibt der Kritikpunkt, dass ihre Ergebnisse ohne Einschränkungen nur für junge Arbeitnehmer und für die USA gültig sind, was die argumentative Reichweite dieser Analyse stark relativiert. Noch uneindeutiger wird das Bild, wenn man die Metaanalyse von Doucouliagos und Stanley hinzuzieht, die sogar eher einen positiven Effekt von Mindestlöhnen konstatieren. Insgesamt kann somit auch vonseiten der empirischen Forschung, trotz des umfangreichen Werkes von Neumark und Wascher, keine eindeutige Aussage zum Beschäftigungseffekt von Mindestlöhnen getroffen werden.

Eines allerdings zeigt die Empirie: Die Diskussion dreht sich letztendlich um die Frage, ob Mindestlöhne einen kleinen negativen, einen kleinen positiven oder gar keinen Beschäftigungseffekt haben. Dies mag aus Sicht der Ökonometrie und für die theoretische Debatte ein ernstzunehmender Streitpunkt sein, für die politische Diskussion ist diese akademische Debatte jedoch weniger relevant. Falls Mindestlöhne tatsächlich mit Beschäftigungsverlusten einhergehen, so kann unter Einbezug der Ergebnisse von Doucouliagos und Stanley davon ausgegangen werden, dass diese relativ gering sind. Sie sollten deshalb in der Erörterung über die Einführung eines Mindestlohnes nicht überbewertet werden, wenn andere wichtige Ziele mit einem Mindestlohn erreicht werden können. Aufgrund des deutschen Institutionsgefüges erscheint die Einführung eines Mindestlohnes hierzulande ohnehin vergleichsweise risikoarm. Und selbst die Studie von Neumark und Wascher, die als Mindestlohngegner zu verorten sind, lässt den Schluss zu, dass ein Mindestlohn sich in Deutschland positiv oder zumindest weniger negativ auswirken wird als in den USA oder Großbritannien. Letzteres hat den Praxistest angetreten und vor etwas mehr als zehn Jahren erstmals einen flächendeckenden Mindestlohn eingeführt.
Was letztlich dazu beigetragen hat, dass das Thema entpolitisiert wurde. Sowohl der Einführungsprozess wie auch die späteren Erhöhungen des Mindestlohnes in Großbritannien wurden wissenschaftlich begleitet. Die Wissenschaftler konnten keinerlei oder nur wenige Hinweise auf negative Beschäftigungseffekte finden (Metcalf 2007, S. 1ff.). Mit diesem Verfahren könnte Großbritannien beispielgebend sein, um eventuelle Risiken bei der Einführung eines Mindestlohnes zu verhindern und einen ideologisch motivierten Schlagabtausch zu unterbinden.

Zwar führen hiesige Kritiker zu Recht an, dass sich die Erfahrungen mit dem Mindestlohn aufgrund der unterschiedlichen Systeme nicht von Großbritannien auf Deutschland übertragen lassen (Raddatz/Wolf 2007, S. 11f.). Entgegen der Voraussage der Kritiker ist jedoch zu erwarten, dass die Effekte eines Mindestlohns in Deutschland aufgrund des regulierten Arbeitsmarktes sogar nochmals positiver ausfallen würden als in Großbritannien.

Insgesamt sind - und das ist das entscheidende Ergebnis dieses Beitrags - die Risiken eines mit Bedacht eingeführten Mindestlohnes für die Beschäftigung in Deutschland als gering einzustufen, sodass das Hauptargument der Mindestlohngegner, die deutliche Beschäftigungsverluste prognostizieren, nicht sticht. Die Befürworter eines Mindestlohnes haben dessen Chancen ausführlich dargestellt. Beispielsweise könnte ein Mindestlohn neben der Bekämpfung des Niedriglohnsektors in Deutschland und der Verringerung der Lohnspreizung nach unten helfen, den deutschen Binnenkonsum anzuregen und so zur Beseitigung der innereuropäischen Ungleichgewichte und der deutschen Exportabhängigkeit beitragen. Wägt man die als geringfügig erscheinenden Risiken eines Mindestlohnes gegen die potenziellen Chancen ab, so sollte die Diskussion um die Frage, $o b$ ein flächendeckender Mindestlohn eingeführt werden soll, umschwenken in eine Debatte darüber, wie dies am besten vollzogen werden kann. Anstatt in ideologischen Grabenkämpfen zu verharren und Simulationen auf Grundlagen unsicherer Annahmen zu erstellen, wäre es besser, wenn sich auch die Wissenschaft an diesem Prozess beteiligen und die politischen Entscheidungsträger beraten würde, wie ein funktionierender Mindestlohn für Deutschland zu gestalten wäre. 


\section{LITERATUR}

Bartsch, K. (2007): Gesamtwirtschaftliche Wirkung der Einführung eines gesetzlichen Mindestlohnes in Deutschland auf der Basis der Konzeption der Dienstleistungsgewerkschaft ver.di. Eine aktualisierte Simulationsstudie mit dem makroökonometrischen Deutschlandmodell LAPROSIM Version 10.32. Gutachten im Auftrag des ver.di - Bundesvorstandes, Neuendorf

Belchamber, G. (2004): Minimum wages and youth employment, in: International Labour Organization (Hrsg.): Policy proposals for decent work and employment for young people, Labour Education 3, S. 29-37 Bhaskar, V./Manning, A./To, T. (2002): Oligopsony and Monopsonistic Competition in Labor Markets, in: The Journal of Economic Perspectives 2, S. 155-174

Brown, C./Gilroy, C./Kohen, A. (1982): The Effect of The Minimum Wage on Employment and Unemployment, in: Journal of Economic Literature 2, S. 487-528

Card, D./Krueger, A. (1995): Myth and Measurement: The New Economics of the Minimum Wage, Princeton 1995

Dolado, J./Kramarz, F./Machin, S./Manning, A./Margolis, D./Teulings, C./Saint-Paul, G./Keen, M. (1996): The Economic Impact of Minimum Wages in Europe, in: Economic Policy 23, S. 319-372 Doucouliagos, H./Stanley, T. (2009): Publication Selection Bias in Minimum-Wage Research? A Meta-Regression Analysis, in: British Journal of Industrial Relations 2, S. 406-428

Fox, L. (2006): Minimum wage trends: Understanding past and contemporary research, Economic Policy Institute, EPI Briefing Paper 178 Herr, H. (2002): Wages, Employment and Prices: An Analysis of the Relationship between Wage Level, Wage Structure, Minimum Wages and Employment and Prices, Business Institute Berlin, School of Economics and Law, Working Paper 15, Berlin

Herr, H./Kanzandziska, M./Mahnkopf-Praprotnik, S. (2009): The theoretical debate about minimum wages, Global Labour University, Paper 6

Knabe, A./Schöb, R. (2008): Minimum Wage Incidence: The Case of Germany, CESifo, Working Paper 2432
Metcalf, D. (2007): Why has the British National Minimum Wage had little or No Impact on Employment?, Centre for Economic Performance, London School of Economics and Political Science, CEP Discussion Paper 781 Müller, K. (2009): How robust are simulated Employment Effects of a Legal Minimum Wage in Germany?, DIW Discussion Paper 900, Berlin Neumark D./Wascher, W. (1998): Is the Time-Series Evidence on Minimum Wage Effects Contaminated by Publication Bias?, in: Economic Inquiry 3, S. 458-470

Neumark, D./Wascher, W. (2004): Minimum Wages, Labor Market Institutions and Youth Employment: A Cross-National Analysis, in: Industrial and Labor Relations Review 2, S. 223-248

Neumark, D./Wascher, W. (2007): Minimum Wages and Employment, Forschungsinstitut zur Zukunft der Arbeit, IZA Discussion Paper 2570, Bonn

Neumark/D., Wascher, W. (2008): Minimum Wages, Cambridge Pereira, S. (2003): The Impact of Minimum Wages on Youth Employment in Portugal, Research Centre for Economic Policy, Research Memorandum 0004, London

Prasch, R. (2000): Reassessing the Labor Supply Curve, in: Journal of Economic 3, S. 679-692

Ragacs, C. (2002): Warum Mindestlöhne die Beschäftigung nicht reduzieren müssen: Ein Literaturüberblick, Wirtschaftsuniversität Wien, Working Paper 19

Ragacs, C. (2004): Minimum Wages and Employment: Static and Dynamic Non-Market-Clearing Equilibrium Models, New York et al. Raddatz, G./Wolf, S. (2007): Irrglaube Mindestlöhne. Trügerische Hoffnung, zerstörte Beschäftigungschancen, Stiftung Marktwirtschaft, Argumente zu Marktwirtschaft und Politik 99, Berlin

Sinn, H. (2007): Der dümmste Spruch des Jahres. Jeder muss von seiner Hände Arbeit leben können? Wer so denkt, der verwechselt Wunsch und Wirklichkeit, in: Süddeutsche Zeitung vom 28. Dezember Zavodny, M. (1998): Why Minimum Wage Hikes May Not Reduce Employment, in: Economic Review, Federal Reserve Bank of Atlanta 2, S. $18-28$ 